\title{
Dihadron interference fragmentation functions in proton-proton collisions
}

\author{
Alessandro Bacchetta* \\ Institut für Theoretische Physik, Universität Regensburg, D-93040 Regensburg, Germany \\ Marco Radic $\dagger$ \\ Dipartimento di Fisica Nucleare e Teorica, Università di Pavia, and \\ Istituto Nazionale di Fisica Nucleare, Sezione di Pavia, I-27100 Pavia, Italy
}

\begin{abstract}
We study the production of hadron pairs in proton-proton collisions, selecting pairs with large total transverse momentum with respect to the beam, and small relative transverse momentum, i.e., belonging to a single jet with large transverse momentum. We describe the process in terms of dihadron fragmentation functions. We consider the production of one pair in polarized collisions (with one transversely polarized proton) and the production of two pairs in unpolarized collisions. In the first case, we discuss how to observe the quark transversity distribution in connection with a specific class of dihadron fragmentation functions, named interference fragmentation functions. In the second case, we suggest how to determine the latter and also how to observe linearly polarized gluons.

PACS numbers: $13.88+\mathrm{e}, 13.85 . \mathrm{Hd}, 13.87 . \mathrm{Fh}$
\end{abstract}

\section{INTRODUCTION}

Spin measurements in high-energy collisions of hadrons can be a powerful and versatile way to investigate the dynamics of quarks and gluons. For instance, several measurements of Single-Spin Asymmetries (SSA) with polarized protons have delivered unexpected results (like the observation of large $A_{N}$ asymmetries by the FNAL E704 [1] and, more recently, by the STAR 2] collaborations), which cannot be justified in the context of perturbative QCD at the partonic level [3]. Unless invoking subleading-twist effects [4, 5], these large asymmetries can be explained by allowing partons both to have an intrinsic transverse momentum [6] and to undergo final-state interactions (which prevents the application of constraints by time-reversal symmetry, leading to the so-called T-odd distribution and fragmentation functions). This interpretation has been further strengthened by recent measurements of SSA with lepton beams by the HERMES 7, 8, 9, 10 and the SMC 11] collaborations, as well as of beam spin asymmetries by the CLAS collaboration [12]. Remarkably, these results can be interpreted as effects due to the orbital angular momentum of partons inside the parent hadron $13,14,15$.

SSA are important not only to study T-odd mechanisms, but also because they can be used as analyzing powers of the quark spin and they allow the measurement of quantities otherwise inaccessible. The most renowned example is the transversity distribution $h_{1}$ [16], a leading-twist parton density that describes the distribution of transversely polarized quarks inside transversely polarized hadrons, an essential piece of information to describe the partonic spin structure of hadrons [17, 18]. Being related to a helicity flipping mechanism (since helicity and chirality coincide at leading twist, it is usually named as a chiral-odd distribution), it is suppressed in inclusive Deep-Inelastic Scattering (DIS) because it requires another chiral-odd partner.

The idea of accessing transversity at leading twist in nucleon-nucleon collisions has been extensively discussed in the literature. The simplest option is Drell-Yan leptoproduction with two transversely polarized protons [16, 17, 19, 20, 21], where the needed chiral-odd partner is the transversity distribution of the corresponding antiquark. This option seems not promising at operating experimental facilities, because the probability of having a transversely polarized antiquark in a transversely polarized proton is suppressed 22, 23]. The situation should be more favorable in the future High Energy Storage Ring at GSI (GSI HESR) [24, 25, 26, 27], where (polarized) antiprotons will be produced. Another option is to consider a semi-inclusive process. For example, if transversely polarized hyperons are produced in collisions where one of the protons is transversely polarized, $h_{1}$ appears in connection with the fragmentation function $H_{1}$ 28, 29]. Alternatively, when a single unpolarized hadron (e.g., a pion) is inclusively produced in hadronic collisions, $h_{1}$ is convoluted with the chiral-odd and T-odd Collins function $H_{1}^{\perp}[30$.

*Electronic address: alessandro.bacchetta@physik.uni-regensburg.de

$\dagger$ Electronic address: radici@pv.infn.it 
The nonperturbative mechanism encoded in $H_{1}^{\perp}$, commonly known as Collins effect, is based on the correlation between the transverse polarization $\mathbf{S}_{T}$ of the fragmenting quark and the orientation of the hadron production plane via the mixed product $\mathbf{S}_{T} \cdot \mathbf{k} \times \mathbf{P}_{h}$, where $\mathbf{k}$ and $\mathbf{P}_{h}$ are the quark and hadron 3-momenta, respectively. Because of residual interactions occurring inside the jet, the T-odd Collins function is sensitive to the phase difference originating from the interference of different production channels. As a consequence, the transverse polarization of the quark influences the azimuthal distribution of the detected pions, producing the observed SSA. Without such residual interactions, the T-odd $H_{1}^{\perp}$ vanishes and the azimuthal distribution of hadrons is perfectly symmetric. But also an intrinsic transverse component of $\mathbf{k}$ is necessary for the Collins effect to survive. In fact, in collinear approximation (i.e., assuming $\mathbf{k}$ and $\mathbf{P}_{h}$ to be parallel) the mixed product vanishes and so does the related SSA.

The need of including the relative transverse momentum between quarks and hadrons brings about several complications: it is much harder to provide factorization proofs 31, to verify the universality of the functions involved [32, 33, 34, 35, 36], and to study their evolution equations [37, 38, 39, 40]. Moreover, for the considered SSA in proton-proton collisions the transverse-momentum dependent elementary cross section gets a suppression factor as an inverse power of the hard scale of the process [41, 42]. Finally, the rich structure of the cross section allows for other competing mechanisms leading to the same SSA, such as the Sivers effect [3], the Qiu-Sterman effect [4], and maybe more [4]. A lively discussion is ongoing in this field in order to correctly interpret the available data for both hadronic collisions and semi-inclusive DIS (see, for example, Refs. 42, 45, 46, 47, 48, 49]).

It has been pointed out already that selecting a more exclusive channel in the final state, where two unpolarized hadrons are detected inside the same jet, is obviously more challenging from the experimental point of view, but it represents a more convenient theoretical situation [50]. ${ }^{1}$ In particular, two vectors are available: the center-of-mass (cm) momentum of the pair, $P_{h}=P_{1}+P_{2}$, and its relative momentum $R=\left(P_{1}-P_{2}\right) / 2$. Therefore, even after integrating the fragmentation functions upon $\mathbf{P}_{h T}$, the intrinsic transverse component of $P_{h}$ with respect to the jet axis, it is still possible to relate the transverse polarization of the fragmenting quark to the transverse component of the relative momentum, $\mathbf{R}_{T}$, via the mixed product $\mathbf{S}_{T} \cdot \mathbf{R} \times \mathbf{P}_{h}$ [53, 54, 55]. For the lepton-induced production of two unpolarized hadrons (e.g., two pions) in semi-inclusive DIS, the generated SSA at leading twist is free from the problems mentioned above about the Collins effect, since the center of mass of the hadron pair is traveling collinear with the jet axis and, thus, collinear factorization is preserved. Indeed, the transversity $h_{1}$ can be coupled to a new class of (chiral-odd and T-odd) fragmentation functions, the so-called Interference Fragmentation Functions (IFF) [56, 57], that can be extracted independently in the corresponding process $e^{+} e^{-} \rightarrow\left(h_{1} h_{2}\right)_{\text {jet }_{C}}\left(h_{1} h_{2}\right)_{j e t_{D}} X[58$, [59], where now two pairs of leading hadrons are detected in each back-to-back jet. Measurements of IFF in semi-inclusive DIS and $e^{+} e^{-}$are under way by the HERMES and BELLE collaborations, respectively, and could be performed by the COMPASS and BABAR collaborations, too.

In this paper, we extend the study of IFF and inclusive two-hadron production to the proton-proton collision case (see also Ref. [60]). In Sect. [II we describe the process $p p^{\uparrow} \rightarrow\left(h_{1} h_{2}\right)_{j e t} X$, where one proton is transversely polarized and one hadron pair is detected inside a jet. We show that only one source of SSA survives at leading twist without any suppression of the hard elementary cross section: it involves the convolution $f_{1} \otimes h_{1} \otimes H_{1}^{\varangle}$, containing the usual unpolarized distribution $f_{1}$, the transversity $h_{1}$ and the IFF $H_{1}^{\varangle}$. No other mechanism is active and the theoretical situation is very clean.

In Sect. III we study the unpolarized collision $p p \rightarrow\left(h_{1} h_{2}\right)_{\text {jet }_{C}}\left(h_{1} h_{2}\right)_{\text {jet }_{D}} X$, where two hadron pairs in separate jets are detected. Two leading-twist Fourier components arise in the azimuthal orientation of the two planes (each one containing one hadron pair) with respect to the scattering plane. One term offers the possibility of observing for the first time the effect of gluon linear polarization in fragmentation processes. The other one is proportional to the convolution $f_{1} \otimes f_{1} \otimes H_{1}^{\varangle} \otimes H_{1}^{\varangle}$; hence, it can be used to measure the unknown IFF $H_{1}^{\varangle}$.

Our work demonstrates how in (polarized) proton-proton collisions it is possible in principle to determine at the same time the transversity distribution $h_{1}$ and the IFF $H_{1}^{\varangle}$, without having to resort to $e^{+} e^{-}$annihilation. The same formalism can be applied to collisions involving (un)polarized antiprotons. Therefore, both processes can be studied not only at experiments such as STAR and PHENIX at RHIC, but also at the future ones being planned at the above mentioned GSI HESR. This one and other conclusions are expanded in Sect. IV]

\section{PRODUCTION OF A SINGLE PAIR}

We consider first the process $A+B \rightarrow\left(C_{1} C_{2}\right)_{C}+X$, where two protons (with momenta $P_{A}, P_{B}$, and spin vectors $\left.S_{A}, S_{B}\right)$ collide, and two unpolarized hadrons $C_{1}, C_{2}$, are inclusively detected inside the same jet $C$. The outgoing

1 Theoretical studies on factorization and evolution of dihadron fragmentation functions have recently appeared [51, 52]. 
hadrons have masses $M_{C 1}$ and $M_{C 2}$, invariant mass $M_{C}$ and momenta $P_{C 1}$ and $P_{C 2}$. It is convenient to introduce the vectors $P_{C}=P_{C 1}+P_{C 2}$ and $R_{C}=\left(P_{C 1}-P_{C 2}\right) / 2$, the total and relative momenta of the pair, respectively (they correspond to the general definition of $P_{h}$ and $R$ in the Introduction). The angle $\theta_{C}$ is the polar angle in the pair's center of mass between the direction of emission (which happens to be back-to-back in this frame) and the direction of $\mathbf{P}_{C}$ in any other frame [57]. The intrinsic transverse component of $P_{C}$ with respect to the jet axis, i.e. $\mathbf{P}_{C T}$, is integrated over and, consequently, $\mathbf{P}_{C}$ is taken parallel to the jet axis itself. The component of $P_{C}$ perpendicular to the beam direction (defined by $\mathbf{P}_{A}$ ) will be denoted as $\mathbf{P}_{C \perp} .^{2}$ Its modulus will serve as the hard scale of the process and it is assumed to be much bigger than the masses of the colliding hadrons and of $M_{C}$. Our analysis is valid only at leading order in $1 /\left|\mathbf{P}_{C \perp}\right|$, i.e. at leading twist.

The cross section for this process can be written, in analogy with single hadron production [42, 61, as

$$
\begin{array}{r}
\frac{d \sigma}{d \eta_{C} d\left|\mathbf{P}_{C \perp}\right| d \cos \theta_{C} d M_{C}^{2} d \phi_{R_{C}} d \phi_{S_{A}} d \phi_{S_{B}}}=2\left|\mathbf{P}_{C \perp}\right| \sum_{a, b, c, d} \frac{1}{4} \sum_{\left(\text {all } \chi^{\prime} \mathrm{s}\right)} \int \frac{d x_{a} d x_{b} d z_{c}}{4 \pi^{2} z_{c}^{2}} \Phi_{a}^{\prime}\left(x_{a}, S_{A}\right)_{\chi_{a}^{\prime} \chi_{a}} \Phi_{b}^{\prime}\left(x_{b}, S_{B}\right)_{\chi_{b}^{\prime} \chi_{b}} \\
\frac{1}{16 \pi \hat{s}^{2}} \hat{M}_{\chi_{c}, \chi_{d} ; \chi_{a}, \chi_{b}} \hat{M}_{\chi_{a}^{\prime}, \chi_{b}^{\prime} ; \chi_{c}^{\prime} \chi_{d}^{\prime}} \Delta_{c}^{\prime}\left(z_{c}, \cos \theta_{C}, M_{C}^{2}, \phi_{R_{C}}\right)_{\chi_{c}^{\prime} \chi_{c}} \delta_{\chi_{d}^{\prime} \chi_{d}} \hat{s} \delta(\hat{s}+\hat{t}+\hat{u}),
\end{array}
$$

where $\eta_{C}$ is the pseudorapidity of the hadron pair, defined with respect to $\mathbf{P}_{A}$.

The azimuthal angles are defined in the hadronic center of mass as follows (see also Fig. 1)

$$
\begin{array}{rlrl}
\cos \phi_{S_{A}} & =\frac{\left(\hat{\mathbf{P}}_{A} \times \mathbf{P}_{C}\right)}{\left|\hat{\mathbf{P}}_{A} \times \mathbf{P}_{C}\right|} \cdot \frac{\left(\hat{\mathbf{P}}_{A} \times \mathbf{S}_{A}\right)}{\left|\hat{\mathbf{P}}_{A} \times \mathbf{S}_{A}\right|}, & \sin \phi_{S_{A}}=\frac{\left(\mathbf{P}_{C} \times \mathbf{S}_{A}\right) \cdot \hat{\mathbf{P}}_{A}}{\left|\hat{\mathbf{P}}_{A} \times \mathbf{P}_{C}\right|\left|\hat{\mathbf{P}}_{A} \times \mathbf{S}_{A}\right|}, \\
\cos \phi_{S_{B}}=\frac{\left(\hat{\mathbf{P}}_{B} \times \mathbf{P}_{C}\right)}{\left|\hat{\mathbf{P}}_{B} \times \mathbf{P}_{C}\right|} \cdot \frac{\left(\hat{\mathbf{P}}_{B} \times \mathbf{S}_{B}\right)}{\left|\hat{\mathbf{P}}_{B} \times \mathbf{S}_{B}\right|}, & \sin \phi_{S_{B}}=\frac{\left(\mathbf{P}_{C} \times \mathbf{S}_{B}\right) \cdot \hat{\mathbf{P}}_{B}}{\left|\hat{\mathbf{P}}_{B} \times \mathbf{P}_{C}\right|\left|\hat{\mathbf{P}}_{B} \times \mathbf{S}_{B}\right|}, \\
\cos \phi_{R_{C}}=\frac{\left(\hat{\mathbf{P}}_{C} \times \mathbf{P}_{A}\right)}{\left|\hat{\mathbf{P}}_{C} \times \mathbf{P}_{A}\right|} \cdot \frac{\left(\hat{\mathbf{P}}_{C} \times \mathbf{R}_{C}\right)}{\left|\hat{\mathbf{P}}_{C} \times \mathbf{R}_{C}\right|}, & \sin \phi_{R_{C}}=\frac{\left(\mathbf{P}_{A} \times \mathbf{R}_{C}\right) \cdot \hat{\mathbf{P}}_{C}}{\left|\hat{\mathbf{P}}_{C} \times \mathbf{P}_{A}\right|\left|\hat{\mathbf{P}}_{C} \times \mathbf{R}_{C}\right|},
\end{array}
$$

where $\hat{\mathbf{P}}=\mathbf{P} /|\mathbf{P}|$.

The partons involved in the elementary scattering have momenta $p_{a}=x_{a} P_{A}, p_{b}=x_{b} P_{B}$, and $p_{c}=P_{C} / z_{c}$. The indices $\chi$ 's refer to the chiralities/helicities of the partons. The partonic hard amplitudes $\hat{M}$ can be taken from Ref. [62] and are written in terms of the partonic Mandelstam variables $\hat{s}, \hat{t}, \hat{u}$, which are related to the external ones by

$$
\hat{s}=x_{a} x_{b} s, \quad \hat{t}=\frac{x_{a}}{z_{c}} t, \quad \hat{u}=\frac{x_{b}}{z_{c}} u .
$$

Conservation of momentum at the partonic level implies that

$$
\hat{s} \delta(\hat{s}+\hat{t}+\hat{u})=z_{c} \delta\left(z_{c}+\frac{x_{a} t+x_{b} u}{x_{a} x_{b} s}\right) \stackrel{\text { c.m.s. }}{=} z_{c} \delta\left(z_{c}-\frac{\left|\mathbf{P}_{C \perp}\right|}{\sqrt{s}} \frac{x_{a} e^{-\eta_{C}}+x_{b} e^{\eta_{C}}}{x_{a} x_{b}}\right) \equiv z_{c} \delta\left(z_{c}-\bar{z}_{c}\right) .
$$

Therefore, Eq. (11) can be written as

$$
\begin{aligned}
\frac{d \sigma}{d \eta_{C} d\left|\mathbf{P}_{C \perp}\right| d \cos \theta_{C} d M_{C}^{2} d \phi_{R_{C}} d \phi_{S_{A}} d \phi_{S_{B}}}= & 2\left|\mathbf{P}_{C \perp}\right| \sum_{a, b, c, d} \frac{1}{4} \sum_{\left(\text {all } \chi^{\prime} \mathrm{s}\right)} \int \frac{d x_{a} d x_{b}}{4 \pi^{2} z_{c}} \Phi_{a}^{\prime}\left(x_{a}, S_{A}\right)_{\chi_{a}^{\prime} \chi_{a}} \Phi_{b}^{\prime}\left(x_{b}, S_{B}\right)_{\chi_{b}^{\prime} \chi_{b}} \\
& \frac{1}{16 \pi \hat{s}^{2}} \hat{M}_{\chi_{c}, \chi_{d} ; \chi_{a}, \chi_{b}} \hat{M}_{\chi_{a}^{\prime}, \chi_{b}^{\prime} ; \chi_{c}^{\prime} \chi_{d}^{\prime}} \Delta_{c}^{\prime}\left(\bar{z}_{c}, \cos \theta_{C}, M_{C}^{2}, \phi_{R_{C}}\right)_{\chi_{c}^{\prime} \chi_{c}} \delta_{\chi_{d}^{\prime} \chi_{d}}
\end{aligned}
$$

where $z_{c}$ is fixed to $\bar{z}_{c}$ by Eq. (6).

The building blocks of Eq. (7) are the following. The correlators $\Phi^{\prime}$ describe the distribution, inside each parent hadron $A$ or $B$, of the two partons entering the elementary vertex and carrying a fraction $x_{a}$ or $x_{b}$, respectively, of

\footnotetext{
${ }^{2}$ In the following, for any vector $V$ pertinent to a hadron $h$ we will use the index $T$ to mean the transverse component of $V$ with respect to the direction of the hadron 3-momentum $\mathbf{P}_{h}$, such that $\mathbf{V}_{T} \cdot \mathbf{P}_{h}=0 ; V$ can be the hadron polarization vector $S_{h}$ (fom which the index $T$ is sometimes used also to mean the transverse polarization), the momentum $p$ of a parton inside $h$, etc. With the index $\perp$ we mean the transverse component of $V$ with respect to the incident beam direction identified by $\mathbf{P}_{A}$.
} 




FIG. 1: Description of the kinematics for the proton-proton annihilation into a single pair in a jet.

the hadron momentum. Parton and corresponding hadron momenta are taken to be parallel, since intrinsic relative transverse components are integrated over. When the partons are quarks, then we have at leading twist and in the quark helicity basis [63, 64]

$$
\Phi_{a}^{\prime}\left(x_{a}, S_{A}\right)_{\chi_{a}^{\prime} \chi_{a}}=\left(\begin{array}{cc}
f_{1}\left(x_{a}\right)+S_{A L} g_{1}\left(x_{a}\right) & \left|\mathbf{S}_{A T}\right| e^{-i \phi_{S_{A}}} h_{1}\left(x_{a}\right) \\
\left|\mathbf{S}_{A T}\right| e^{i \phi_{S_{A}}} h_{1}\left(x_{a}\right) & f_{1}\left(x_{a}\right)-S_{A L} g_{1}\left(x_{a}\right)
\end{array}\right)
$$

where we omitted flavor indices; $f_{1}$ and $g_{1}$ are the unpolarized and helicity distributions of quark $a$ in proton $A$. The correlator is written in the helicity basis where $\mathbf{P}_{A}$ defines the $\hat{z}$ axis, with $S_{A L}$ and $\left|\mathbf{S}_{A T}\right|$ indicating the parallel and transverse components of the polarization $\mathbf{S}_{A}$ with respect to $\mathbf{P}_{A}$, and the $\hat{x}$ axis is oriented along $\mathbf{P}_{C \perp}$. For quark $b$ in hadron $B$ we have a similar correlator by replacing $a, A$ with $b, B$; consequently, the $\hat{z}$ axis is now pointing along $\mathbf{P}_{B}$ and the helicity $S_{B L}$ is considered positive or negative with respect to this axis.

When the partons are gluons, the correlator reads [65, 66$]$

$$
\Phi^{\prime}\left(x_{a}, S_{A}\right)_{\chi_{a}^{\prime} \chi_{a}}=\left(\begin{array}{cc}
G\left(x_{a}\right)+S_{A L} \Delta G\left(x_{a}\right) & 0 \\
0 & G\left(x_{a}\right)-S_{A L} \Delta G\left(x_{a}\right)
\end{array}\right)
$$

where $G(x), \Delta G(x)$, are the unpolarized and helicity gluon distributions; an analogous formula holds for gluon $b$ in hadron $B$. To our purposes, it is very important to note that in a spin- $\frac{1}{2}$ target there is no gluon transversity because of the mismatch in the helicity change [67]; hence, the off-diagonal elements in Eq. (9) are vanishing.

The last ingredient in Eq. (7) is the correlator $\Delta_{c}^{\prime}$ describing the fragmentation of the parton $c$ into two hadrons. Up to leading twist, the correlator for a fragmenting quark $c$ reads 57

$$
\Delta_{c}^{\prime}\left(z_{c}, \cos \theta_{C}, M_{C}^{2}, \phi_{R_{C}}\right)_{\chi_{c}^{\prime} \chi_{c}}=\frac{1}{4 \pi}\left(\begin{array}{cc}
D_{1}^{c} & i e^{i \phi_{R_{C}}} \frac{\left|\mathbf{R}_{C}\right|}{M_{C}} \sin \theta_{C} H_{1}^{\varangle c} \\
-i e^{-i \phi_{R_{C}}} \frac{\left|\mathbf{R}_{C}\right|}{M_{C}} \sin \theta_{C} H_{1}^{\varangle c} & D_{1}^{c}
\end{array}\right),
$$

where the fragmentation functions inside the matrix depend on $z_{c}, \cos \theta_{C}, M_{C}^{2}$, and

$$
\left|\mathbf{R}_{C}\right|=\frac{1}{2} \sqrt{M_{C}^{2}-2\left(M_{1}^{2}+M_{2}^{2}\right)+\left(M_{1}^{2}-M_{2}^{2}\right)^{2} / M_{C}^{2}} .
$$

The correlator is written in the helicity basis by choosing this time the $\hat{z}$ axis along $\mathbf{P}_{C}$ and the $\hat{x}$ axis to point along the component of $\mathbf{P}_{A}$ orthogonal to $\mathbf{P}_{C}$ (see Fig. 1).

The fragmentation functions inside the correlator (10) can be expanded in relative partial waves using the basis of Legendre polinomials through the $\cos \theta_{C}$ dependence [57]; truncating the expansion at $L=1$, we have

$$
\begin{aligned}
D_{1}\left(z_{c}, \cos \theta_{C}, M_{C}^{2}\right) & \rightarrow D_{1, o o}\left(z_{c}, M_{C}^{2}\right)+D_{1, o l}\left(z_{c}, M_{C}^{2}\right) \cos \theta_{C}+D_{1, l l}\left(z_{c}, M_{C}^{2}\right) \frac{1}{4}\left(3 \cos ^{2} \theta_{C}-1\right), \\
\sin \theta_{C} H_{1}^{\varangle}\left(z_{c}, \cos \theta_{C}, M_{C}^{2}\right) & \rightarrow H_{1, o t}^{\varangle}\left(z_{c}, M_{C}^{2}\right) \sin \theta_{C}+H_{1, l t}^{\varangle}\left(z_{c}, M_{C}^{2}\right) \sin \theta_{C} \cos \theta_{C} .
\end{aligned}
$$


Since the fragmentation functions at leading twist are probability densities, the double-index notation refers to the polarization state in the center of mass of the pair for each separate probability amplitude; namely, $D_{1, o o}$ describes the decay probability into two hadrons in a relative $L=0$ wave, while $D_{1, o l}$ describes the interference between the amplitudes for the decay into a $L=0$ pair and a $L=1$ pair "longitudinally" polarized along $\mathbf{P}_{C}$ in its $\mathrm{cm}$ frame (hence, proportional to $\cos \theta_{C}$ ). Similarly, $H_{1, o t}^{\varangle}$ describes the interference between a $L=0$ pair and a "transversely" polarized $L=1$ pair (proportional to $\sin \theta_{C}$ ), while $D_{1, l l}$ and $H_{1, l t}^{\varangle}$ refer to interferences between different polarization components of $L=1$ pairs.

In general, the invariant-mass dependence of the fragmentation functions is unknown. However, we know that the $p$-wave contribution originates essentially from a spin- 1 resonance (e.g., the $\rho$ meson in two-pion production). ${ }^{3}$ Therefore, we can expect the pure $p$-wave fragmentation functions, $D_{1, l l}$ and $H_{1, l t}^{\varangle}$, to display the invariant mass dependence typical of the resonance (e.g., a Breit-Wigner peaked at the $\rho$ mass). The fragmentation function $D_{1, o o}$ contains both $s$ and $p$-wave contributions, and should therefore look as the superposition of the resonance peak and of a continuum background. For what concerns the interference terms, $D_{1, o l}$ and $H_{1, o t}^{\triangleleft}$, since both $s$ and $p$ channels have to be present, they should be sizeable only in the neighborhood of the resonance, possibly with a sign change at the position of the resonance peak [54].

When parton $c$ is a gluon, the correlator $\Delta_{c}^{\prime}$ describes the yet unexplored fragmentation of a gluon into two hadrons. Due to angular momentum conservation, we know that for gluons the off-diagonal elements of $\Delta_{c}^{\prime}$ can contain only pure $L=1$ contributions from the fragmentation of a gluon into a spin- 1 resonance 69|. The correlator at leading twist can be obtained in analogy to the case of gluon distributions in spin-1 targets [6, ,77]:

$$
\Delta_{c}^{\prime}\left(z_{c}, \cos \theta_{C}, M_{C}^{2}, \phi_{R_{C}}\right)_{\chi_{c}^{\prime} \chi_{c}}=\frac{1}{4 \pi}\left(\begin{array}{cc}
\hat{G}^{c} & i e^{2 i \phi_{R_{C}}} \frac{\left|\mathbf{R}_{C}\right|^{2}}{M_{C}^{2}} \sin ^{2} \theta_{C} \delta \hat{G}^{\varangle c} \\
-i e^{-2 i \phi_{R_{C}}} \frac{\left|\mathbf{R}_{C}\right|^{2}}{M_{C}^{2}} \sin ^{2} \theta_{C} \delta \hat{G}^{\varangle c} & \hat{G}^{c}
\end{array}\right) .
$$

The function $\hat{G}$ describes the decay of an unpolarized gluon into two unpolarized hadrons. Its partial-wave expansion is the same as that of $D_{1}$ in Eq. (12). The function $\delta \hat{G}^{\varangle}$ describes the decay into two unpolarized hadrons of a transversely polarized gluon. ${ }^{4}$ We choose the symbol $\delta \hat{G}^{\varangle}$ for the new function to indicate a transverse gluon fragmentation function that needs an explicit dependence upon the relative transverse momentum between the two hadrons. Unfortunately, $\delta \hat{G}^{\varangle}$ cannot appear in connection with the quark transversity distribution $h_{1}$ because of the mismatch in the units of helicity flip between a spin- $\frac{1}{2}$ and a spin-1 objects, leading to the $\exp \left(i \phi_{R_{C}}\right)$ and $\exp \left(2 i \phi_{R_{C}}\right)$ dependences in Eq. (8) and Eq. (13), respectively. It can be coupled to the gluon transversity $\delta G$, which is defined only in targets with spin greater than $\frac{1}{2}[19$, 76]. If we truncate the partial-wave expansion to $L=1$, due to angularmomentum conservation $\delta \hat{G}^{\varangle}$ contains only the $L=1$ contribution and reduces to the fragmentation of a gluon into a vector meson [69]. Consequently, it is possible to predict that it will follow the invariant-mass shape of a spin-1 resonance. The $\theta_{C}$ dependence is given only by the $\sin ^{2} \theta_{C}$ prefactor, typical of a transversely polarized resonance.

When in the proton-proton collision one of the two protons is transversely polarized, namely the process $p p^{\uparrow} \rightarrow$ $\left(h_{1} h_{2}\right)_{j e t} X$, the most interesting SSA is

$$
A_{N}\left(\eta_{C},\left|\mathbf{P}_{C \perp}\right|, \cos \theta_{C}, M_{C}^{2}, \phi_{R_{C}}, \phi_{S_{B}}\right)=\frac{d \sigma^{\uparrow}-d \sigma^{\downarrow}}{d \sigma^{\uparrow}+d \sigma^{\downarrow}} \equiv \frac{d \sigma_{U T}}{d \sigma_{U U}}
$$

where the proton transversity distribution $h_{1}$ can be observed at leading twist in connection with the IFF $H_{1}^{\varangle}$. The longitudinal spin asymmetry displays no new features compared to the case where only one hadron is produced.

For the unpolarized cross section in the denominator of Eq. (14) (integrated over $\phi_{S_{A}}$ ), we have

$$
d \sigma_{U U}=2\left|\mathbf{P}_{C \perp}\right| \sum_{a, b, c, d} \int \frac{d x_{a} d x_{b}}{8 \pi^{2} z_{c}} f_{1}^{a}\left(x_{a}\right) f_{1}^{b}\left(x_{b}\right) \frac{d \hat{\sigma}_{a b \rightarrow c d}}{d \hat{t}} D_{1}^{c}\left(\bar{z}_{c}, \cos \theta_{C}, M_{C}^{2}\right)
$$

\footnotetext{
3 The $p$-wave contributions are identical to vector-meson fragmentation functions, which have been studied in several articles in the context of different experiments $68,69,70,71,72,73,74,75]$.

4 The "transverse" polarization of a gluon can be defined in strict analogy to the quark case. Indicating the positive/negative helicity states along a certain $\hat{z}$ axis by $| \pm\rangle$, we introduce the states $|\uparrow\rangle=1 / \sqrt{2}(|+\rangle+|-\rangle)$ and $|\downarrow\rangle=i / \sqrt{2}(|+\rangle-|-\rangle)$. They correspond to linearly polarized gluons along the two independent directions orthogonal to $\hat{z}$, i.e. the $\hat{x}$ and $\hat{y}$ axis [19]. However, to keep a uniform notation and to avoid confusion between linear and longitudinal polarization, we prefer to talk about transverse polarization states and to use the superscript $\uparrow$ when necessary (note that a different notation was used, e.g., in Ref. [78]).
} 
Here and in the following expressions, it is understood that when the parton is a gluon, we need to make the replacements $f_{1} \rightarrow G$ and $D_{1} \rightarrow \hat{G}$. The unpolarized elementary cross sections are well known [62]. For convenience, we rewrite them in Eqs. A.2 A.5 in the Appendix.

For the transversely polarized cross section in the numerator of Eq. (14) (integrated over $\phi_{S_{A}}$ ), we have

$$
\begin{aligned}
d \sigma_{U T}= & 2\left|\mathbf{P}_{C \perp}\right| \sum_{a, b, c, d}\left|\mathbf{S}_{B T}\right| \sin \left(\phi_{S_{B}}-\phi_{R_{C}}\right) \int \frac{d x_{a} d x_{b}}{8 \pi^{2} z_{c}} f_{1}^{a}\left(x_{a}\right) h_{1}^{b}\left(x_{b}\right) \frac{d \Delta \hat{\sigma}_{a b \uparrow \rightarrow c^{\uparrow} d}}{d \hat{t}} \\
& \times \frac{\left|\mathbf{R}_{C}\right|}{M_{C}} \sin \theta_{C} H_{1}^{\varangle c}\left(\bar{z}_{c}, \cos \theta_{C}, M_{C}^{2}\right) \\
\approx & 2\left|\mathbf{P}_{C \perp}\right| \sum_{a, b, c, d} \frac{\left|\mathbf{R}_{C}\right|}{M_{C}}\left|\mathbf{S}_{B T}\right| \sin \left(\phi_{S_{B}}-\phi_{R_{C}}\right) \int \frac{d x_{a} d x_{b}}{8 \pi^{2} z_{c}} f_{1}^{a}\left(x_{a}\right) h_{1}^{b}\left(x_{b}\right) \frac{d \Delta \hat{\sigma}_{a b^{\uparrow} \rightarrow c^{\uparrow} d}}{d \hat{t}} \\
& \times \sin \theta_{C}\left(H_{1, o t}^{\varangle c}\left(\bar{z}_{c}, M_{C}^{2}\right)+\cos \theta_{C} H_{1, l t}^{\varangle c}\left(\bar{z}_{c}, M_{C}^{2}\right)\right) .
\end{aligned}
$$

The elementary cross sections with transversely polarized partons $b$ and $c$ correspond to

$$
\frac{d \Delta \hat{\sigma}_{a b^{\uparrow} \rightarrow c^{\uparrow} d}}{d \hat{t}} \equiv \frac{1}{16 \pi \hat{s}^{2}} \frac{1}{4} \sum_{\left(\text {all } \chi^{\prime} \mathrm{s}\right)} \hat{M}_{\chi_{c}, \chi_{d} ; \chi_{a}, \chi_{b}} \hat{M}_{\chi_{a},-\chi_{b} ;-\chi_{c}, \chi_{d}}^{*}
$$

They describe the cross section for the case when quark $b$ is transversely polarized in a direction forming an azimuthal angle $\phi_{S_{b}}$ around $\mathbf{P}_{B}$ and the transverse polarization of quark $c$ forms the same azimuthal angle $\phi_{S_{c}}=\phi_{S_{b}}$ around $\mathbf{P}_{C}\left(\phi_{S_{b}}\right.$ and $\phi_{S_{c}}$ are defined analogously to $\phi_{S_{B}}$ and $\phi_{R_{C}}$, respectively - see Eqs. (3) and (4) and Fig. (1). We list them explicitly in Eqs. A.7 A.10) in the Appendix (see also Ref. 79]).

It is possible to integrate the cross sections over $\cos \theta_{C}$ to obtain

$$
\begin{aligned}
& d \sigma_{U U}=2\left|\mathbf{P}_{C \perp}\right| \sum_{a, b, c, d} \int \frac{d x_{a} d x_{b}}{4 \pi^{2} z_{c}} f_{1}^{a}\left(x_{a}\right) f_{1}^{b}\left(x_{b}\right) \frac{d \hat{\sigma}_{a b \rightarrow c d}}{d \hat{t}} D_{1, o o}\left(\bar{z}_{c}, M_{C}^{2}\right), \\
& d \sigma_{U T}=2\left|\mathbf{P}_{C \perp}\right| \sum_{a, b, c, d} \frac{\left|\mathbf{R}_{C}\right|}{M_{C}}\left|\mathbf{S}_{B T}\right| \sin \left(\phi_{S_{B}}-\phi_{R_{C}}\right) \int \frac{d x_{a} d x_{b}}{16 \pi z_{c}} f_{1}^{a}\left(x_{a}\right) h_{1}^{b}\left(x_{b}\right) \frac{d \Delta \hat{\sigma}_{a b b^{\uparrow} \rightarrow c^{\uparrow} d}}{d \hat{t}} H_{1, o t}^{\varangle c}\left(\bar{z}_{c}, M_{C}^{2}\right) .
\end{aligned}
$$

Eqs. (16) and (19) are the most relevant results of this Section and a few comments are in order. First of all, we obtain a formula reminiscent of the original one proposed by Tang, Eq. (8) in Ref. 60] . However, there are some crucial differences: in Ref. [60] the dependence on the azimuthal angles is different, the connection to the external variables (rapidity and transverse momentum of the pair) is not made clear, and the behavior in the invariant mass is factorized out of the IFF, which is a model-dependent statement. Finally, a couple of differences in the elementary cross sections are pointed out in the Appendix. Our asymmetries are also analogous to the ones for the process $p p^{\uparrow} \rightarrow \Lambda^{\uparrow} X$ discussed in Refs. [28, 29]. In that case, however, the asymmetry is proportional to $\cos \left(\phi_{S_{B}}-\phi_{S_{\Lambda}}\right)$ (with $\phi_{S_{\Lambda}}$ being the azimuthal angle of the transverse spin of the $\Lambda$, defined analogously to $\phi_{R_{C}}$ ): the transverse spin of the quark is directly transferred to the transverse spin of the $\Lambda$, while here it is connected to $\mathbf{R}_{C T}$ via the mixed product entailed in the T-odd $H_{1}^{\varangle}$, which implies an extra $\frac{\pi}{2}$ rotation. Finally, our asymmetry can be related to that occurring in two-hadron production in DIS [56, [57], by replacing $f_{1}\left(x_{a}\right)$ with $\delta\left(1-x_{a}\right)$, using the elementary cross section for $l q^{\uparrow} \rightarrow q^{\uparrow} l$ and taking into account the fact that the final parton $d$ is also observed, so that $x_{b}$ and $z_{c}$ are fixed according to Eq. (21) of next Sect. [II with $z_{d}=1$. In Refs. [56, 57], the asymmetry depends on $\sin \left(\phi_{S_{B}}+\phi_{R_{C}}\right)$ simply because the azimuthal angles are defined in a different way with respect to the present work.

Quantitative estimates of these asymmetries are possible either by using models for the IFF [54, 56, 80] or by saturating their positivity bounds [57]. Measurements of IFF are under way at HERMES (semi-inclusive DIS) and at BELLE [81] ( $e^{+} e^{-}$annihilation). Experimental results at different energies can be related through the same DGLAP equations applicable to the fragmentation function $H_{1}$ [82]..$^{5}$ However, as we shall see in the next Section, IFF can be measured independently in the very same proton-proton collisions analyzed so far.

\footnotetext{
${ }^{5}$ Note that we deal with functions that depend explicitly on the (limited) relative transverse momentum of the hadron pair. On the contrary, the evolution equations studied in Refs. [51, 52] apply to dihadron fragmentation functions integrated over the relative transverse momentum of the hadron pair.
} 


\section{PRODUCTION OF TWO PAIRS}

As already anticipated in Sect. [1] the simultaneous detection of two hadron pairs belonging to two separate jets allows the extraction of the specific IFF, $H_{1}^{\varangle}$, that occurs coupled to the transversity $h_{1}$ in the corresponding production of a single hadron pair in one jet (see previous Sect. II).

The definition of the momenta and angles of the second hadron pair is done in complete analogy to the first pair, just replacing all indices $c, C$ with $d, D$. The correlation function $\Delta_{d}^{\prime}$ is obtained in the same way. The generic expression for the cross section is

$$
\begin{aligned}
& \frac{d \sigma}{d \eta_{C} d\left|\mathbf{P}_{C \perp}\right| d \cos \theta_{C} d M_{C}^{2} d \phi_{R_{C}} d \eta_{D} d\left|\mathbf{P}_{D \perp}\right| d \cos \theta_{D} d M_{D}^{2} d \phi_{R_{D}} d \phi_{S_{A}} d \phi_{S_{B}}} \\
& =2 \sum_{a, b, c, d} \frac{1}{4} \sum_{\left(\text {all } \chi^{\prime} \mathrm{s}\right)} \int \frac{d x_{a}}{4 \pi^{2}} \Phi_{a}^{\prime}\left(x_{a}, S_{A}\right)_{\chi_{a}^{\prime} \chi_{a}} \bar{x}_{b} \Phi_{b}^{\prime}\left(\bar{x}_{b}, S_{B}\right)_{\chi_{b}^{\prime} \chi_{b}} \frac{1}{16 \pi \hat{s}^{2}} \hat{M}_{\chi_{c}, \chi_{d} ; \chi_{a}, \chi_{b}} \hat{M}_{\chi_{a}^{\prime}, \chi_{b}^{\prime} ; \chi_{c}^{\prime} \chi_{d}^{\prime}} \\
& \quad \Delta_{c}^{\prime}\left(\bar{z}_{c}, \cos \theta_{C}, M_{C}^{2}, \phi_{R_{C}}\right)_{\chi_{c}^{\prime} \chi_{c}} \Delta_{d}^{\prime}\left(\bar{z}_{d}, \cos \theta_{D}, M_{D}^{2}, \phi_{R_{D}}\right)_{\chi_{d}^{\prime} \chi_{d}}
\end{aligned}
$$

with

$$
\bar{z}_{c} \stackrel{\text { c.m.s. }}{=} \frac{\left|\mathbf{P}_{C \perp}\right|}{\sqrt{s}} \frac{e^{\eta_{C}}+e^{\eta_{D}}}{x_{a}}, \quad \quad \bar{z}_{d} \stackrel{\text { c.m.s. }}{=} \frac{\left|\mathbf{P}_{D \perp}\right|}{\sqrt{s}} \frac{e^{\eta_{C}}+e^{\eta_{D}}}{x_{a}}, \quad \bar{x}_{b} \stackrel{\text { c.m.s. }}{=} x_{a} e^{-\eta_{C}} e^{-\eta_{D}} .
$$

The above relations are obtained from momentum conservation at the partonic level.

For unpolarized proton-proton collisions, the main observable is the unpolarized cross section (integrated over the angles $\phi_{S_{A}}$ and $\left.\phi_{S_{B}}\right)$

$$
d \sigma_{U U}=\mathcal{A}+\cos \left(\phi_{R_{C}}-\phi_{R_{D}}\right) \mathcal{B}+\cos \left(2 \phi_{R_{C}}-2 \phi_{R_{D}}\right) \mathcal{C}
$$

The function $\mathcal{A}$ is given by

$$
\begin{aligned}
\mathcal{A}= & \sum_{a, b, c, d} \int \frac{d x_{a}}{8 \pi^{2}} f_{1}^{a}\left(x_{a}\right) \bar{x}_{b} f_{1}^{b}\left(\bar{x}_{b}\right) \frac{d \hat{\sigma}_{a b \rightarrow c d}}{d \hat{t}} D_{1}^{c}\left(\bar{z}_{c}, \cos \theta_{C}, M_{C}^{2}\right) D_{1}^{d}\left(\bar{z}_{d}, \cos \theta_{D}, M_{D}^{2}\right) \\
\approx & \sum_{a, b, c, d} \int \frac{d x_{a}}{8 \pi^{2}} f_{1}^{a}\left(x_{a}\right) \bar{x}_{b} f_{1}^{b}\left(\bar{x}_{b}\right) \frac{d \hat{\sigma}_{a b \rightarrow c d}}{d \hat{t}} \\
& \times\left(D_{1, o o}^{c}\left(\bar{z}_{c}, M_{C}^{2}\right)+D_{1, o l}^{c}\left(\bar{z}_{c}, M_{C}^{2}\right) \cos \theta_{C}+D_{1, l l}^{c}\left(\bar{z}_{c}, M_{C}^{2}\right) \frac{1}{4}\left(3 \cos ^{2} \theta_{C}-1\right)\right) \\
& \times\left(D_{1, o o}^{d}\left(\bar{z}_{d}, M_{D}^{2}\right)+D_{1, o l}^{d}\left(\bar{z}_{d}, M_{D}^{2}\right) \cos \theta_{D}+D_{1, l l}^{d}\left(\bar{z}_{d}, M_{D}^{2}\right) \frac{1}{4}\left(3 \cos ^{2} \theta_{D}-1\right)\right),
\end{aligned}
$$

where the elementary cross sections $d \hat{\sigma}_{a b \rightarrow c d}$ are given by Eqs. A.2 A.5. In other words, $\mathcal{A}$ is the analogue of Eq. (15) for the production of two hadron pairs in separate jets. When the parton is a gluon, we need to make the replacements $f_{1} \rightarrow G$ and $D_{1} \rightarrow \hat{G}$.

The function $\mathcal{B}$ is given by

$$
\begin{aligned}
\mathcal{B}= & \sum_{a, b, c, d} \int \frac{d x_{a}}{8 \pi^{2}} f_{1}^{a}\left(x_{a}\right) \bar{x}_{b} f_{1}^{b}\left(\bar{x}_{b}\right) \frac{d \Delta \hat{\sigma}_{a b \rightarrow c^{\uparrow} d^{\uparrow}}}{d \hat{t}} \frac{\left|\mathbf{R}_{C}\right|}{M_{C}} \sin \theta_{C} H_{1}^{\varangle c}\left(\bar{z}_{c}, \cos \theta_{C}, M_{C}^{2}\right) \frac{\left|\mathbf{R}_{D}\right|}{M_{D}} \sin \theta_{D} H_{1}^{\varangle d}\left(\bar{z}_{d}, \cos \theta_{D}, M_{D}^{2}\right) \\
\approx & \sum_{a, b, c, d} \int \frac{d x_{a}}{8 \pi^{2}} f_{1}^{a}\left(x_{a}\right) \bar{x}_{b} f_{1}^{b}\left(\bar{x}_{b}\right) \frac{d \Delta \hat{\sigma}_{a b \rightarrow c^{\uparrow} d^{\uparrow}}}{d \hat{t}} \frac{\left|\mathbf{R}_{C}\right|}{M_{C}} \sin \theta_{C}\left(H_{1, o t}^{\varangle c}\left(\bar{z}_{c}, M_{C}^{2}\right)+\cos \theta_{C} H_{1, l t}^{\varangle c}\left(\bar{z}_{c}, M_{C}^{2}\right)\right) \\
& \times \frac{\left|\mathbf{R}_{D}\right|}{M_{D}} \sin \theta_{D}\left(H_{1, o t}^{\varangle d}\left(\bar{z}_{d}, M_{D}^{2}\right)+\cos \theta_{D} H_{1, l t}^{\varangle d}\left(\bar{z}_{d}, M_{D}^{2}\right)\right),
\end{aligned}
$$

with partons $c$ and $d$ being necessarily quarks and with the relevant partonic cross sections being

$$
\frac{d \Delta \hat{\sigma}_{a b \rightarrow c^{\uparrow} d^{\uparrow}}}{d \hat{t}} \equiv \frac{1}{16 \pi \hat{s}^{2}} \frac{1}{4} \sum_{\left(\text {all } \chi^{\prime} \mathrm{s}\right)} \hat{M}_{\chi_{c}, \chi_{d} ; \chi_{a}, \chi_{b}} \hat{M}_{\chi_{a}, \chi_{b} ;-\chi_{c},-\chi_{d}}^{*} .
$$

They are explicitly listed in Eqs. A.12 A.14 in the Appendix. 
Finally, the function $\mathcal{C}$ is

$$
\begin{aligned}
\mathcal{C}= & \sum_{a, b, c, d} \int \frac{d x_{a}}{8 \pi^{2}} f_{1}^{a}\left(x_{a}\right) \bar{x}_{b} f_{1}^{b}\left(\bar{x}_{b}\right) \frac{d \Delta \hat{\sigma}_{a b \rightarrow g^{\uparrow} g^{\uparrow}}}{d \hat{t}} \\
& \times \frac{\left|\mathbf{R}_{C}\right|^{2}}{M_{C}^{2}} \sin ^{2} \theta_{C} \delta \hat{G}^{\varangle c}\left(\bar{z}_{c}, \cos \theta_{C}, M_{C}^{2}\right) \frac{\left|\mathbf{R}_{D}\right|^{2}}{M_{D}^{2}} \sin ^{2} \theta_{D} \delta \hat{G}^{\varangle d}\left(\bar{z}_{d}, \cos \theta_{C}, M_{D}^{2}\right) \\
\approx & \sum_{a, b, c, d} \int \frac{d x_{a}}{8 \pi^{2}} f_{1}^{a}\left(x_{a}\right) \bar{x}_{b} f_{1}^{b}\left(\bar{x}_{b}\right) \frac{d \Delta \hat{\sigma}_{a b \rightarrow g^{\uparrow} g^{\uparrow}}}{d \hat{t}} \\
& \times \frac{\left|\mathbf{R}_{C}\right|^{2}}{M_{C}^{2}} \sin ^{2} \theta_{C} \delta \hat{G}^{\varangle c}\left(\bar{z}_{c}, M_{C}^{2}\right) \frac{\left|\mathbf{R}_{D}\right|^{2}}{M_{D}^{2}} \sin ^{2} \theta_{D} \delta \hat{G}^{\varangle d}\left(\bar{z}_{d}, M_{D}^{2}\right),
\end{aligned}
$$

where the nonvanishing elementary cross sections are given in Eq. A.15 in the Appendix.

After integrating upon the angles $\theta_{C}$ and $\theta_{D}$, we obtain

$$
d \sigma_{U U}=\mathcal{A}^{\prime}+\cos \left(\phi_{R_{C}}-\phi_{R_{D}}\right) \mathcal{B}^{\prime}+\cos \left(2 \phi_{R_{C}}-2 \phi_{R_{D}}\right) \mathcal{C}^{\prime},
$$

where

$$
\begin{aligned}
\mathcal{A}^{\prime} & =\int_{0}^{\pi} d \theta_{C} \sin \theta_{C} \int_{0}^{\pi} d \theta_{D} \sin \theta_{D} \mathcal{A} \\
& =\sum_{a, b, c, d} \int \frac{d x_{a}}{2 \pi^{2}} f_{1}^{a}\left(x_{a}\right) \bar{x}_{b} f_{1}^{b}\left(\bar{x}_{b}\right) \frac{d \hat{\sigma}_{a b \rightarrow c d}}{d \hat{t}} D_{1, o o}\left(\bar{z}_{c}, M_{C}^{2}\right) D_{1, o o}\left(\bar{z}_{d}, M_{D}^{2}\right), \\
\mathcal{B}^{\prime} & =\int_{0}^{\pi} d \theta_{C} \sin \theta_{C} \int_{0}^{\pi} d \theta_{D} \sin \theta_{D} \mathcal{B} \\
& =\sum_{a, b, c, d} \int \frac{d x_{a}}{32} f_{1}^{a}\left(x_{a}\right) \bar{x}_{b} f_{1}^{b}\left(\bar{x}_{b}\right) \frac{d \Delta \hat{\sigma}_{a b \rightarrow c^{\uparrow} d^{\uparrow}}}{d \hat{t}} \frac{\left|\mathbf{R}_{C}\right|}{M_{C}} H_{1, o t}^{\varangle c}\left(\bar{z}_{c}, M_{C}^{2}\right) \frac{\left|\mathbf{R}_{D}\right|}{M_{D}} H_{1, o t}^{\varangle d}\left(\bar{z}_{d}, M_{D}^{2}\right), \\
\mathcal{C}^{\prime} & =\int_{0}^{\pi} d \theta_{C} \sin \theta_{C} \int_{0}^{\pi} d \theta_{D} \sin \theta_{D} \mathcal{C} \\
& =2 \sum_{a, b, c, d} \int \frac{d x_{a}}{9 \pi^{2}} f_{1}^{a}\left(x_{a}\right) \bar{x}_{b} f_{1}^{b}\left(\bar{x}_{b}\right) \frac{d \Delta \hat{\sigma}_{a b \rightarrow g^{\uparrow} g^{\uparrow}}}{d \hat{t}} \frac{\left|\mathbf{R}_{C}\right|^{2}}{M_{C}^{2}} \delta \hat{G}^{\varangle c}\left(\bar{z}_{c}, M_{C}^{2}\right) \frac{\left|\mathbf{R}_{D}\right|^{2}}{M_{D}^{2}} \delta \hat{G}^{\varangle d}\left(\bar{z}_{d}, M_{D}^{2}\right) .
\end{aligned}
$$

The above expressions can be related to what has been obtained for the case of $e^{+} e^{-}$annihilation [58], by replacing $f_{1}(x)$ with $\delta(1-x)$ and using the elementary cross section for $e^{+} e^{-} \rightarrow q^{\uparrow} \bar{q}^{\uparrow}$ (clearly no gluon contribution is present). Once again, the apparent difference in the resulting angular dependence is due solely to a different definition of the azimuthal angles.

Both functions $\mathcal{B}$ and $\mathcal{C}$ (or $\mathcal{B}^{\prime}$ and $\mathcal{C}^{\prime}$ ) are interesting. The first one contains two interference fragmentation functions $H_{1}^{\varangle}$, one for each hadron pair: measuring the $\cos \left(\phi_{R_{C}}-\phi_{R_{D}}\right)$ asymmetry of the cross section for the $p p \rightarrow\left(h_{1} h_{2}\right)_{j e t_{C}}\left(h_{1} h_{2}\right)_{j e t_{D}} X$ process allows the extraction of $H_{1}^{\varangle}$ and, in turn, of the transversity $h_{1}$ from the $d \sigma_{U T}$ asymmetry described in the previous Section. The second observable, $\mathcal{C}$ (or $\mathcal{C}^{\prime}$ ), describes the fragmentation of two transversely (linearly) polarized gluons into two transversely (linearly) polarized spin-1 resonances.

\section{CONCLUSIONS}

Understanding the polarization of partons inside hadrons is a fundamental goal in order to describe the partonic structure of the hadrons themselves. At present, the main missing piece of information is represented by the quark transversity distribution, $h_{1}$, a leading-twist parton density that describes the distribution of transversely polarized quarks inside transversely polarized hadrons. Its chiral-odd nature has prevented it from being measured in the simplest elementary processes like inclusive DIS. Several alternative strategies have been suggested in the literature, among which the most popular ones are transversely polarized Drell-Yan [16] and the Collins effect [30] in semiinclusive DIS with transversely polarized targets.

Selecting a more exclusive final state with two detected hadrons inside the same jet could be a more convenient option. Even when the center of mass of the two hadrons is assumed to move collinear with the jet axis, the transverse component of the relative momentum of the two hadrons (with respect to the jet axis, or equivalently with respect 
to the center-of-mass direction) is still available to build a single-spin asymmetry that singles out $h_{1}$ at leading twist via an interference fragmentation function, $H_{1}^{\varangle}[56]$. The asymmetry described by $H_{1}^{\varangle}$ is related to the azimuthal position of the hadron-pair plane with respect to the scattering plane. All distribution and fragmentation functions can be integrated over intrinsic transverse momenta, making it simpler to deal with issues such as evolution equations, factorization and universality [36]. The comparison between hadron-hadron collisions, semi-inclusive DIS [50] and $e^{+} e^{-}$annihilation [58] becomes therefore simpler than for the Collins effect.

In this paper, we have applied the formalism of interference fragmentation functions to proton-proton collisions. We have shown that in the production of one hadron pair in collisions with one transversely polarized proton, it is possible to isolate the convolution $f_{1} \otimes h_{1} \otimes H_{1}^{\varangle}$, involving the usual momentum distribution $f_{1}$, through the measurement of the asymmetry of the cross section in the azimuthal orientation of the pair around its center-of-mass momentum. In the production of two hadron pairs in two separate jets in unpolarized collisions, it is possible to isolate the convolution $f_{1} \otimes f_{1} \otimes H_{1}^{\varangle} \otimes H_{1}^{\varangle}$, through the measurement of the asymmetry of the cross section in the azimuthal orientation of the two pairs around their center-of-mass momenta. Since no distribution and fragmentation functions with an explicit transverse-momentum dependence are required, there is no need to consider suppressed contributions in the elementary cross sections included in the convolutions and the discussed asymmetries remain at leading twist. Therefore proton-proton collisions offer a unique possibility to measure simultaneously the transversity distribution $h_{1}$, and the interference fragmentation function $H_{1}^{\varangle}$.

Finally, we have also shown that unpolarized proton-proton collisions into two hadron pairs (basically into two spin-1 resonances) can also provide novel information on the role of gluon linear polarization.

Our formalism can be applied also to collisions involving (polarized) antiprotons. Therefore, it can be used by experimental collaborations working on existing machines with polarized proton beams (like STAR and PHENIX at RHIC), but also with polarized antiprotons at GSI HESR. Unpolarized collisions into two hadron pairs can be studied also at Tevatron and LHC. In the future, we hope that it will be possible to compare experimental results in hadron-hadron collisions with those in semi-inclusive DIS and $e^{+} e^{-}$annihilation and perform a global analysis of dihadron fragmentation functions.

\section{Acknowledgments}

Discussions with M. Stratmann, B. Jäger are gratefully acknowledged. The work of A. B. has been supported by the Alexander von Humboldt Foundation.

\section{APPENDIX: ELEMENTARY CROSS SECTIONS}

We list here the unpolarized partonic cross sections

$$
\begin{array}{ccc}
\frac{d \hat{\sigma}_{a b \rightarrow c d}}{d \hat{t}} \equiv \frac{1}{16 \pi \hat{s}^{2}} \frac{1}{4} \sum_{\left(\text {all } \chi^{\prime} \mathrm{s}\right)} \hat{M}_{\chi_{c}, \chi_{d} ; \chi_{a}, \chi_{b}} \hat{M}_{\chi_{a}, \chi_{b} ; \chi_{c} \chi_{d}}, \\
\frac{d \hat{\sigma}_{q q \rightarrow q q}}{d \hat{t}}=\frac{4 \pi \alpha_{s}^{2}}{9}\left(\frac{\hat{s}^{4}+\hat{t}^{4}+\hat{u}^{4}}{\hat{s}^{2} \hat{t}^{2} \hat{u}^{2}}-\frac{8}{3 \hat{t} \hat{u}}\right), & \frac{d \hat{\sigma}_{q q^{\prime} \rightarrow q^{\prime} q}}{d \hat{t}}=\frac{4 \pi \alpha_{s}^{2}}{9}\left(\frac{\hat{s}^{2}+\hat{t}^{2}}{\hat{s}^{2} \hat{u}^{2}}\right), \\
\frac{d \hat{\sigma}_{q \bar{q} \rightarrow q^{\prime} \bar{q}^{\prime}}}{d \hat{t}}=\frac{4 \pi \alpha_{s}^{2}}{9}\left(\frac{\hat{t}^{2}+\hat{u}^{2}}{\left.\hat{s}^{4}\right),}\right. & \frac{d \hat{\sigma}_{q \bar{q} \rightarrow q \bar{q}}}{d \hat{t}}=\frac{4 \pi \alpha_{s}^{2}}{9 \hat{s}^{4} \hat{t}^{2}}\left(\hat{s}^{4}+\hat{t}^{4}+\hat{u}^{4}-\frac{8}{3} \hat{s} \hat{t} \hat{u}^{2}\right), \\
\frac{d \hat{\sigma}_{q \bar{q} \rightarrow g g}}{d \hat{t}}=\frac{8 \pi \alpha_{s}^{2}}{3} \frac{\hat{t}^{2}+\hat{u}^{2}}{\hat{s}^{2}}\left(\frac{4}{9 \hat{t} \hat{u}}-\frac{1}{\hat{s}^{2}}\right), & \frac{d \hat{\sigma}_{g q \rightarrow g q}}{d \hat{t}}=\pi \alpha_{s}^{2} \frac{\hat{s}^{2}+\hat{t}^{2}}{\hat{s}^{2}}\left(\frac{1}{\hat{t}^{2}}-\frac{4}{9 \hat{s} \hat{u}}\right), \\
\frac{d \hat{\sigma}_{g g \rightarrow g g}}{d \hat{t}}=\frac{9 \pi \alpha_{s}^{2}}{8} \frac{\left(\hat{s}^{4}+\hat{t}^{4}+\hat{u}^{4}\right)\left(\hat{s}^{2}+\hat{t}^{2}+\hat{u}^{2}\right)}{\hat{s}^{4} \hat{t}^{2} \hat{u}^{2}}, & \frac{d \hat{\sigma}_{g g \rightarrow q \bar{q}}}{d \hat{t}}=\frac{3 \pi \alpha_{s}^{2}}{8} \frac{\hat{t}^{2}+\hat{u}^{2}}{\hat{s}^{2}}\left(\frac{4}{9 \hat{t} \hat{u}}-\frac{1}{\hat{s}^{2}}\right) .
\end{array}
$$

All other nonvanishing cross sections can be obtained from these ones by means of simple crossings.

We define the partonic cross sections differences with transversely polarized partons $b$ and $c$ as

$$
\frac{d \Delta \hat{\sigma}_{a b \uparrow \rightarrow c^{\uparrow} d}}{d \hat{t}} \equiv \frac{1}{16 \pi \hat{s}^{2}} \frac{1}{4} \sum_{\left(\text {all } \chi^{\prime} \mathrm{s}\right)} \hat{M}_{\chi_{c}, \chi_{d} ; \chi_{a}, \chi_{b}} \hat{M}_{\chi_{a},-\chi_{b} ;-\chi_{c}, \chi_{d}}^{*} .
$$


In principle, these are not cross sections, but rather bilinear combinations of amplitudes. However, they correspond to cross sections for specific polarization states of the partons involved. The nonvanishing ones are

$$
\begin{aligned}
& \frac{d \Delta \hat{\sigma}_{q q^{\uparrow} \rightarrow q^{\uparrow} q}}{d \hat{t}}=-\frac{8 \pi \alpha_{s}^{2}}{27 \hat{s}^{2}} \frac{\hat{s}(3 \hat{t}-\hat{u})}{\hat{u}^{2}}, \\
& \frac{d \Delta \hat{\sigma}_{q \bar{q}^{\uparrow} \rightarrow q^{\uparrow} \bar{q}}}{d \hat{t}}=-\frac{8 \pi \alpha_{s}^{2}}{27 \hat{s}^{2}}, \\
& \frac{d \Delta \hat{\sigma}_{g q^{\uparrow} \rightarrow q^{\uparrow} g}}{d \hat{t}}=-\frac{8 \pi \alpha_{s}^{2}}{9 \hat{s}^{2}}\left(1-\frac{9}{4} \frac{\hat{t} \hat{s}}{\hat{u}^{2}}\right), \\
& \frac{d \Delta \hat{\sigma}_{g g^{\uparrow} \rightarrow g^{\uparrow} g}}{d \hat{t}}=\frac{9 \pi \alpha_{s}^{2}}{2 \hat{s}^{2}} \frac{\hat{u}^{2}-\hat{s} \hat{t}}{\hat{u}^{2}} .
\end{aligned}
$$

These cross sections correspond to the results presented in Table 1 of Ref. 79], when the initial and final azimuthal angles of the quarks (as defined in Sec. III) are equal (in the language of that paper, when $A\left(s_{b}, s_{c}\right)=-t / 2$, or equivalently when $\beta=\Phi-\pi / 2$ ). They correspond also to the "transversity dependent" cross sections of Table I of Ref. [60] (to compare the results, $\hat{t}$ and $\hat{u}$ have to be interchanged, since $a$ is the polarized parton in Ref. 60]), except for a factor 2 difference in the last term of the $q \bar{q}^{\uparrow} \rightarrow \bar{q}^{\uparrow} q$ cross section and for the absence in Ref. 60] of the $q \bar{q}^{\uparrow} \rightarrow q^{\uparrow} \bar{q}$ cross section. The last two partonic cross sections are missing in both Refs. [79] and [60]: they are less useful because there is no gluon transversity inside the proton. We present them for completeness and for possible future applications with spin-1 targets. As already clarified in Sec. II we use the transverse gluon polarization states, $|\uparrow\rangle$ and $|\downarrow\rangle$, in place of linear polarization states along the $\hat{x}$ and $\hat{y}$ directions [19] (note that a different notation was used, e.g., in Ref. [8] ).

We introduce the partonic cross section differences for two transversely polarized partons in the final state

$$
\frac{d \Delta \hat{\sigma}_{a b \rightarrow c^{\uparrow} d^{\uparrow}}}{d \hat{t}} \equiv \frac{1}{16 \pi \hat{s}^{2}} \frac{1}{4} \sum_{\left(\text {all } \chi^{\prime} \mathrm{s}\right)} \hat{M}_{\chi_{c}, \chi_{d} ; \chi_{a}, \chi_{b}} \hat{M}_{\chi_{a}, \chi_{b} ;-\chi_{c},-\chi_{d}}^{*} .
$$

When the final-state partons are quarks, we have the following nonvanishing cross sections, to be used in Eq. (24):

$$
\begin{aligned}
\frac{d \Delta \hat{\sigma}_{q q \rightarrow q^{\uparrow} q^{\uparrow}}}{d \hat{t}} & =-\frac{8 \pi \alpha_{s}^{2}}{27 \hat{s}^{2}}, \\
\frac{d \Delta \hat{\sigma}_{q \bar{q} \rightarrow \bar{q}_{T} q_{T}}}{d \hat{t}} & =-\frac{8 \pi \alpha_{s}^{2}}{27 \hat{s}^{2}} \frac{\hat{t}(3 \hat{u}-\hat{s})}{\hat{s}^{2}}, \\
\frac{d \Delta \hat{\sigma}_{g g \rightarrow q^{\uparrow} \bar{q}^{\uparrow}}}{d \hat{t}} & =-\frac{\pi \alpha_{s}^{2}}{3 \hat{s}^{2}}\left(1-\frac{9}{4} \frac{\hat{t} \hat{u}}{\hat{s}^{2}}\right) .
\end{aligned}
$$

When the final-state partons are gluons, we have the following nonvanishing cross sections, to be used in Eq. (26):

$$
\frac{d \Delta \hat{\sigma}_{q \bar{q} \rightarrow g^{\uparrow} g^{\uparrow}}}{d \hat{t}}=-\frac{64 \pi \alpha_{s}^{2}}{27 \hat{s}^{2}}\left(1-\frac{9}{4} \frac{\hat{t} \hat{u}}{\hat{s}^{2}}\right), \quad \frac{d \Delta \hat{\sigma}_{g g \rightarrow g^{\uparrow} g^{\uparrow}}}{d \hat{t}}=\frac{9 \pi \alpha_{s}^{2}}{2 \hat{s}^{2}} \frac{\hat{u}^{2}-\hat{s} \hat{t}}{\hat{s}^{2}} .
$$

[1] D. L. Adams et al. (FNAL-E704), Phys. Lett. B264, 462 (1991).

[2] J. Adams et al. (STAR), Phys. Rev. Lett. 92, 171801 (2004), hep-ex/0310058.

[3] G. L. Kane, J. Pumplin, and W. Repko, Phys. Rev. Lett. 41, 1689 (1978).

[4] J. Qiu and G. Sterman, Phys. Rev. Lett. 67, 2264 (1991).

[5] Y. Kanazawa and Y. Koike, Phys. Lett. B478, 121 (2000), hep-ph/0001021.

[6] M. Anselmino, M. Boglione, and F. Murgia, Phys. Lett. B362, 164 (1995), hep-ph/9503290.

[7] A. Airapetian et al. (HERMES) (2004), hep-ex/0408013.

[8] A. Airapetian et al. (HERMES), Phys. Rev. Lett. 84, 4047 (2000), hep-ex/9910062.

[9] A. Airapetian et al. (HERMES), Phys. Rev. D64, 097101 (2001), hep-ex/0104005.

[10] A. Airapetian et al. (HERMES), Phys. Lett. B562, 182 (2003), hep-ex/0212039.

[11] A. Bravar (Spin Muon), Nucl. Phys. A666, 314 (2000).

[12] H. Avakian (CLAS) (2003), hep-ex/0301005.

[13] S. J. Brodsky, D. S. Hwang, and I. Schmidt, Phys. Lett. B530, 99 (2002), hep-ph/0201296.

[14] A. V. Belitsky, X. Ji, and F. Yuan, Nucl. Phys. B656, 165 (2003), hep-ph/0208038. 
[15] M. Burkardt and D. S. Hwang, Phys. Rev. D69, 074032 (2004), hep-ph/0309072.

[16] J. P. Ralston and D. E. Soper, Nucl. Phys. B152, 109 (1979).

[17] R. L. Jaffe and X. Ji, Phys. Rev. Lett. 67, 552 (1991).

[18] V. Barone and P. G. Ratcliffe, Transverse Spin Physics (World Scientific, River Edge, USA, 2003).

[19] X. Artru and M. Mekhfi, Z. Phys. C45, 669 (1990).

[20] J. L. Cortes, B. Pire, and J. P. Ralston, Z. Phys. C55, 409 (1992).

[21] X. Ji, Phys. Lett. B284, 137 (1992).

[22] O. Martin, A. Schafer, M. Stratmann, and W. Vogelsang, Phys. Rev. D57, 3084 (1998), hep-ph/9710300.

[23] V. Barone, T. Calarco, and A. Drago, Phys. Rev. D56, 527 (1997), hep-ph/9702239.

[24] A. V. Efremov, K. Goeke, and P. Schweitzer, Eur. Phys. J. C35, 207 (2004), hep-ph/0403124.

[25] M. Anselmino, V. Barone, A. Drago, and N. N. Nikolaev (2004), hep-ph/0403114.

[26] PAX Collab., Letter of Intent for Antiproton-Proton Scattering Experiments with Polarization, Spokespersons: F. Rathmannn and P. Lenisa (2003), http://www.fz-juelich.de/ikp/pax/.

[27] M. Radici (2004), invited talk at The $2^{\text {nd }}$ PANDA Physics Workshop, hep-ph/0407085.

[28] D. de Florian, M. Stratmann, and W. Vogelsang, Phys. Rev. Lett. 81, 530 (1998), hep-ph/9802432.

[29] D. de Florian, J. Soffer, M. Stratmann, and W. Vogelsang, Phys. Lett. B439, 176 (1998), hep-ph/9806513.

[30] J. C. Collins, Nucl. Phys. B396, 161 (1993), hep-ph/9208213.

[31] X. Ji, J.-P. Ma, and F. Yuan (2004), hep-ph/0405085.

[32] J. C. Collins, Phys. Lett. B536, 43 (2002), hep-ph/0204004.

[33] A. Metz, Phys. Lett. B549, 139 (2002), hep-ph/0209054.

[34] D. Boer, P. J. Mulders, and F. Pijlman, Nucl. Phys. B667, 201 (2003), hep-ph/0303034.

[35] C. J. Bomhof, P. J. Mulders, and F. Pijlman, Phys. Lett. B596, 277 (2004), hep-ph/0406099.

[36] J. Collins and A. Metz (2004), hep-ph/0408249.

[37] D. Boer, Nucl. Phys. B603, 195 (2001), hep-ph/0102071.

[38] A. A. Henneman, D. Boer, and P. J. Mulders, Nucl. Phys. B620, 331 (2002), hep-ph/0104271.

[39] R. Kundu and A. Metz, Phys. Rev. D65, 014009 (2002), hep-ph/0107073.

[40] A. Idilbi, X. Ji, J.-P. Ma, and F. Yuan (2004), hep-ph/0406302.

[41] M. Anselmino and F. Murgia, Phys. Lett. B442, 470 (1998), hep-ph/9808426.

[42] M. Anselmino, M. Boglione, U. D’Alesio, E. Leader, and F. Murgia (2004), hep-ph/0408356.

[43] D. W. Sivers, Phys. Rev. D41, 83 (1990).

[44] D. Boer, Phys. Rev. D60, 014012 (1999), hep-ph/9902255.

[45] A. V. Efremov, K. Goeke, and P. Schweitzer, Phys. Lett. B568, 63 (2003), hep-ph/0303062.

[46] P. Schweitzer and A. Bacchetta, Nucl. Phys. A732, 106 (2004), hep-ph/0310318.

[47] A. Bacchetta, P. J. Mulders, and F. Pijlman, Phys. Lett. B595, 309 (2004), hep-ph/0405154.

[48] U. D'Alesio and F. Murgia (2004), hep-ph/0408092.

[49] B.-Q. Ma, I. Schmidt, and J.-J. Yang (2004), hep-ph/0409012.

[50] A. Bacchetta and M. Radici, Phys. Rev. D69, 074026 (2004), hep-ph/0311173.

[51] D. de Florian and L. Vanni, Phys. Lett. B578, 139 (2004), hep-ph/0310196.

[52] A. Majumder and X.-N. Wang, Phys. Rev. D70, 014007 (2004), hep-ph/0402245.

[53] J. C. Collins and G. A. Ladinsky (1994), hep-ph/9411444.

[54] R. L. Jaffe, X. Jin, and J. Tang, Phys. Rev. Lett. 80, 1166 (1998), hep-ph/9709322.

[55] A. Bianconi, S. Boffi, R. Jakob, and M. Radici, Phys. Rev. D62, 034008 (2000), hep-ph/9907475.

[56] M. Radici, R. Jakob, and A. Bianconi, Phys. Rev. D65, 074031 (2002), hep-ph/0110252.

[57] A. Bacchetta and M. Radici, Phys. Rev. D67, 094002 (2003), hep-ph/0212300.

[58] D. Boer, R. Jakob, and M. Radici, Phys. Rev. D67, 094003 (2003), hep-ph/0302232.

[59] X. Artru and J. C. Collins, Z. Phys. C69, 277 (1996), hep-ph/9504220.

[60] J. Tang (1998), hep-ph/9807560.

[61] M. Anselmino, M. Boglione, and F. Murgia, Phys. Rev. D60, 054027 (1999), hep-ph/9901442.

[62] R. Gastmans and T. T. Wu, The Ubiquitous photon: Helicity method for QED and QCD, International series of monographs on physics (Clarendon, Oxford, UK, 1990).

[63] R. L. Jaffe and X. Ji, Nucl. Phys. B375, 527 (1992).

[64] A. Bacchetta, M. Boglione, A. Henneman, and P. J. Mulders, Phys. Rev. Lett. 85, 712 (2000), hep-ph/9912490.

[65] R. L. Jaffe and N. Saito, Phys. Lett. B382, 165 (1996), hep-ph/9604220.

[66] P. J. Mulders and J. Rodrigues, Phys. Rev. D63, 094021 (2001), hep-ph/0009343.

[67] R. L. Jaffe (1996), proceedings of the Ettore Majorana International School on the Spin Structure of the Nucleon, Erice, Italy, 3-10 Aug 1995., hep-ph/9602236.

[68] A. V. Efremov and O. V. Teryaev, Sov. J. Nucl. Phys. 36, 140 (1982).

[69] M. Anselmino and P. Kroll, Phys. Rev. D30, 36 (1984).

[70] X. Ji, Phys. Rev. D49, 114 (1994), hep-ph/9307235.

[71] M. Anselmino, M. Boglione, J. Hansson, and F. Murgia, Phys. Rev. D54, 828 (1996), hep-ph/9512379.

[72] M. Anselmino, M. Bertini, F. Caruso, F. Murgia, and P. Quintairos, Eur. Phys. J. C11, 529 (1999), hep-ph/9904205.

[73] A. Bacchetta and P. J. Mulders, Phys. Rev. D62, 114004 (2000), hep-ph/0007120.

[74] Q.-h. Xu, C.-x. Liu, and Z.-t. Liang, Phys. Rev. D63, 111301 (2001), hep-ph/0103267.

[75] Q.-h. Xu and Z.-t. Liang, Phys. Rev. D67, 114013 (2003), hep-ph/0304125. 
[76] R. L. Jaffe and A. Manohar, Phys. Lett. B223, 218 (1989).

[77] A. Schafer, L. Szymanowski, and O. V. Teryaev, Phys. Lett. B464, 94 (1999), hep-ph/9906471.

[78] W. Vogelsang, Acta Phys. Polon. B29, 1189 (1998), hep-ph/9805295.

[79] M. Stratmann and W. Vogelsang, Phys. Lett. B295, 277 (1992).

[80] A. Bianconi, S. Boffi, R. Jakob, and M. Radici, Phys. Rev. D62, 034009 (2000), hep-ph/9907488.

[81] M. Grosse Perdekamp, Nucl. Phys. Proc. Suppl. 105, 71 (2002).

[82] M. Stratmann and W. Vogelsang, Phys. Rev. D65, 057502 (2002), hep-ph/0108241. 